\title{
Acute Promeylocytic Leukemia Presenting as Fatal Intracranial Bleeding in a Rural Setting
}

\author{
Ng Kwang How ${ }^{1}$, Satesh Ramasundram¹, Naganathan Pillai ${ }^{2}$ \\ ${ }^{1}$ Department of Medicine, Segamat Hospital, Johor, Malaysia \\ ${ }^{2}$ Department of Medicine, Monash Medical Segamat Precinct, Johor, Malaysia \\ Email: pillainag@gmail.com
}

How to cite this paper: How, N.K., Ramasundram, S. and Pillai, N. (2020) Acute Promeylocytic Leukemia Presenting as Fatal Intracranial Bleeding in a Rural Setting. Open Access Library Journal, 7: 66006. https://doi.org/10.4236/oalib.1106006

Received: December 16, 2019

Accepted: January 7, 2020

Published: January 10, 2020

Copyright $\odot 2020$ by author(s) and Open Access Library Inc.

This work is licensed under the Creative Commons Attribution International License (CC BY 4.0).

http://creativecommons.org/licenses/by/4.0/

\begin{abstract}
Acute promyelocytic leukemia (APL) is a biologically and clinically distinct variant of acute myeloid leukemia (AML), and accounts for 5 to 20 percent of all AML cases. It is a highly curable hematological malignancy with targeted therapy. Patients usually present with symptoms of the complications, typically bleeding. Intracranial bleeding with APL is rarely seen at the presentation, but more common in relapsed cases. APL is a medical emergency with a high rate of early mortality, usually due to catastrophic bleeding if it is not treated timely. It is vital to start treatment once the diagnosis is suspected without waiting for definite diagnosis. We presented a 35-year-old foreign worker who presented with headache and blurring of vision for 5 days, associated with fever and multiple episodes of vomiting on the day of admission. Subsequently he developed status epilepticus and disseminated bleeding. $\mathrm{He}$ had raised leukocyte, low platelet, prolonged international normalized ratio and prolonged prothrombin time. His computed tomography (CT) brain revealed intracranial hemorrhage. He was intubated and was transfused one cycle of disseminated intravascular coagulation regime. He was referred to intensive care unit and neurosurgical team. Unfortunately, the patient passed away within 5 hours of hospitalization. His urgent peripheral blood film subsequently came back after he passed away and showed increased white blood cell with more than $90 \%$ of abnormal promyelocytes, Auer rods and Faggot cells, suggestive of APL. This case highlights the importance of high index of suspicion; the timely and urgent treatment, which is crucial and might prevent the catastrophic event.
\end{abstract}

\section{Subject Areas}

Hematology

\section{Keywords}

Acute Promyelocytic Leukaemia, Intracranial Bleed, CT Brain, Peripheral Blood Film 


\section{Case Presentation}

A 35 years old Bangladesh man with no known medical illnesses, presented with five days history of headache and blurring of vision, associated with fever and vomiting for more than 10 times on the day of admission. There was no obvious bleeding during the initial assessment at emergency department and he had no history of trauma. On examination, he appeared to be drowsy with the GCS of E3V3M5. His blood pressure was $125 / 72$, heart rate was 80 , Temperature was 37.5 degree Celsius, and glucometer was 7.3. His neurological examination showed upper motor neuron lesions on the right side (hyper-reflexia, hypertonia and up going plantar response). Cardiovascular, respiratory and abdominal examinations were unremarkable. He was initially treated as meningoencephalitis empirically with antibiotics with the differential diagnosis of space occupying lesion and was planned for urgent CT brain. Subsequently, he developed refractory generalized tonic clinic movements and required intubation for airway protection. Post intubation, he started to have spontaneous bleeding from ear, nasal and oral cavity. His full blood count was white blood cells $\left(13.9 \times 10^{3} / \mathrm{ul}\right)$, hemoglobin $(6.1 \mathrm{~g} / \mathrm{dL})$ and platelet $\left(20 \times 10^{9} / \mathrm{L}\right)$. He had prolonged international normalized ratio (1.73) and prolonged thrombin time (19.9). Activated partial thromboplastin time was 35.4 seconds. Renal profile and liver function test were unremarkable. His CT brain revealed left parieto-temporal bleed with intraventricular bleeding extension, midline shift, left cerebral edema and basal cistern effacement with early hydrocephalus (Figure 1). He was immediately referred to intensive care unit and neurosurgical team for further care. One cycle of disseminated intravascular coagulation regime (6 units of cryoprecipitate, 4 units of fresh frozen plasma and 2 units of platelets) was transfused. However, he succumbed to death within 5 hours of hospitalization. His peripheral blood film subsequently came back after he passed away and showed increased WBC with more than $90 \%$ of abnormal promyelocytes, Auer rods and Faggot cells (Figure 2), suggestive of APL.

\section{Introduction}

Acute myeloid leukemia (AML) is a hematological malignancy involving cells committed to the myeloid lineage. AML is classified by the World Health Organization classification based on immunophenotype, clinical features, morphology and genetics. Acute promyelocytic leukemia (APL) is a biologically and clinically distinct variant of AML. APL is currently classified as acute promyelocytic leukemia with $\mathrm{t}(15 ; 17)$ (q24.1; q21.2); PML-RARA in the World Health Organization classification system [1]. The cells from almost all patients have a balanced reciprocal translocation between chromosomes 15 and 17 [2]. It generates a fusion transcript joining the PML (promyelocyte) and RAR- $\alpha$ (retinoic acid receptor- $\alpha$ ) genes [3]. APL accounts for 5 to 20 percent of cases of AML. There is no gender variation. It is not common in the first decade of life. The incidence will increase during early adulthood and is usually diagnosed around 40 


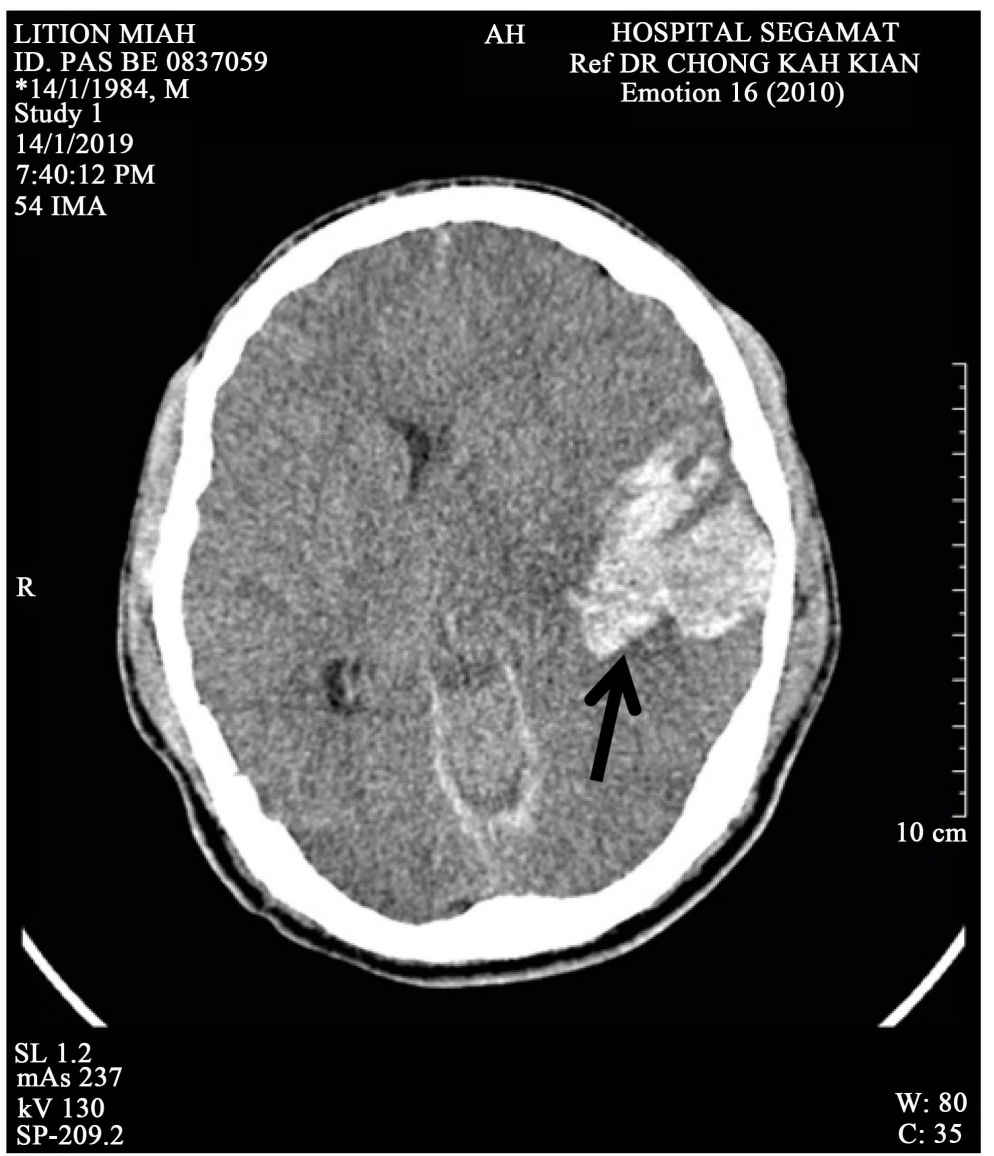

Figure 1. Shows a CT brain with left parieto-temporal bleed, intraventricular bleeding extension, midline shift, left cerebral edema and basal cistern effacement with early hydrocephalus.

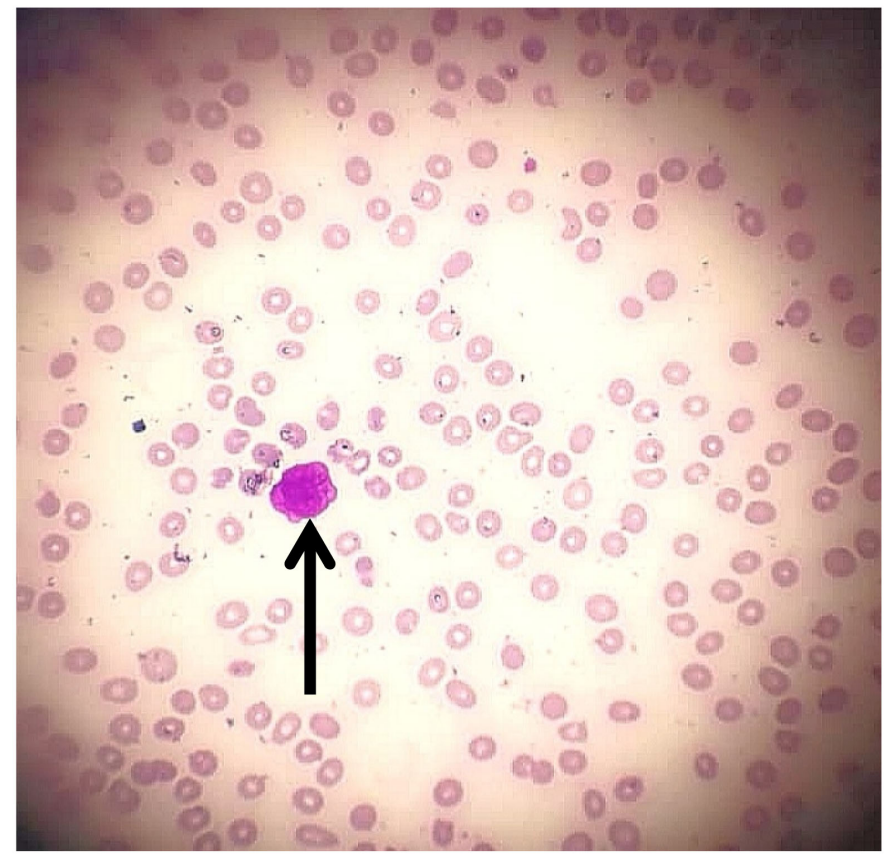

Figure 2. Shows the Peripheral blood film with Auer rod. 
years old. The risk factors include repeated exposure to benzene, cigarette smoking or high levels of radiation.

Patients with APL typically present with symptoms related to complications of pancytopenia, including weakness and easy fatigability, infections of variable severity, and/or hemorrhagic findings such as gingival bleeding, ecchymoses, epistaxis, or menorrhagia. Combinations of these symptoms are common. APL also might present with bleeding secondary to disseminated intravascular coagulation [4]. Central nervous system involvement with APL commonly occurs in relapse, it is rarely seen at presentation, with only six reported cases in the literature [5]. It is a medical emergency with a high rate of early mortality, often due to hemorrhage from a characteristic coagulopathy. Coagulopathy associated with APL is complex and involves both disseminated intravascular coagulation and primary hyper-fibrinolysis and is either present at diagnosis or occurs soon after the initiation of cytotoxic chemotherapy [6].

\section{Diagnosis}

The presumptive diagnosis of APL can usually be made by review of the peripheral blood smear alone or with the bone marrow aspirate and biopsy, in the presence of the characteristic clinical findings [7]. The diagnosis is confirmed by the identification of the PML-RARA fusion gene and or the associated chromosomal translocation [4].

\section{Discussion}

The treatment of patients with APL is a true emergency. It should be started at the earliest suspicion of APL, even before the diagnosis is confirmed by cytogenetic or molecular studies in patients with suspected APL. Very early treatment at the first suspicion of the diagnosis may be one of the few ways in which the early death rate resulting from fatal bleeding can be decreased [8]. Without treatment, APL is the most malignant form of AML, with a median survival of less than one month [9]. The treatment of APL has three stages (remission induction, consolidation and maintenance), in total may span one to two years of treatment [10].

Risk classification is determined based on the white blood cell (WBC) and platelet count. For patient with low- or intermediate-risk disease (WBC < $10,000 / \mu \mathrm{L}$ ), all-trans retinoic acid (ATRA) plus arsenic trioxide (ATO) rather than ATRA plus anthracycline-based chemotherapy is recommended [11]. However, for patient presents with high-risk disease (WBC $\geq 10,000 / \mu \mathrm{L}$ ), ATRA plus anthracycline-based chemotherapy is the most commonly used initial therapy [11]. ATRA is the key component of the therapy regime as it promotes the terminal differentiation of malignant promyelocytes to mature neutrophils. The optimal dose and duration of ATRA regime are not established. The standard dose of ATRA regime is continued until achievement of complete remission by peripheral blood and bone marrow criteria as long as the patient is tolerating it well [12]. 
Approximately 90 percent of patients with newly diagnosed APL will achieve a hematological complete remission with induction therapy. However, without consolidation therapy, virtually all of these patients will relapse. Consolidation therapy is directed at leukemia cells that survived induction therapy but are not detectable by conventional tests [11]. Maintenance therapy decreases the incidence of relapse further and improves disease-free survival for those who have undergone induction with ATRA plus chemotherapy followed by consolidation therapy [11]. For patients with relapsed or refractory disease, allogeneic or autologous haematopoietic cell transplantation plus chemotherapy are able to improve the prognosis and survival.

APL is the first example of a malignant disease that is highly curable with molecularly targeted therapy against its specific genetic abnormality [12]. The cure rate of approximately $80 \%$ to $90 \%$ of patients who survive induction and achieve complete remission can be expected [10] [13]. Early mortality is usually due to catastrophic bleeding.

\section{Conclusion}

Managing APL can be challenging in a rural setting due to the lack of relevant expertise and facilities. Our intention is to alert the health care personnel regarding the possibility of APL in a patient presenting with high WBC, low platelet and fatal bleeding. It also highlights the importance of high index of suspicion, effective communication between clinicians, haematologist and haemato-pathologist which may provide a timely diagnosis and treatment.

\section{Conflicts of Interest}

The authors declare no conflicts of interest regarding the publication of this paper.

\section{References}

[1] Arber, D.A., Orazi, A., Hasserjian, R., et al. (2016) The 2016 Revision to the World Health Organization Classification of Myeloid Neoplasms and Acute Leukemia. Blood, 127, 2391-2405. https://doi.org/10.1182/blood-2016-03-643544

[2] Rowley, J.D., Golomb, H.M. and Dougherty, C. (1977) 15/17 Translocation, a Consistent Chromosomal Change in Acute Promyelocytic Leukaemia. Lancet, 1, 549-550. https://doi.org/10.1016/S0140-6736(77)91415-5

[3] Wang, Z.Y. and Chen, Z. (2008) Acute Promyelocytic Leukemia: From Highly Fatal to Highly Curable. Blood, 111, 2505-2515. https://doi.org/10.1182/blood-2007-07-102798

[4] https://www.uptodate.com/contents/clinical-manifestations-pathologic-features-an d-diagnosis-of-acute-promyelocytic-leukemia-in-adults? source=history widget

[5] Bhuller, K. and Kwan, M. (2011) Acute Promyelocyticleukaemia Presenting with Subarachnoid Haemorrhage and Complicated by Central Nervous System Involvement. Blood Cancer Journal, 1, e25. https://doi.org/10.1038/bcj.2011.21

[6] Mantha, S., Tallman, M.S. and Soff, G.A. (2016) What's New in the Pathogenesis of 
the Coagulopathy in Acute Promyelocytic Leukemia? Curr Opin Hematol, 23, 121-126. https://doi.org/10.1097/MOH.0000000000000221

[7] Bennett, J.M., Catovsky, D., Daniel, M.T., et al. (2000) Hypergranularpromyelocytic Leukemia: Correlation between Morphology and Chromosomal Translocations Including $\mathrm{t}(15 ; 17)$ and $\mathrm{t}(11 ; 17)$. Leukemia, 14, 1197-1200.

https://doi.org/10.1038/sj.leu.2401795

[8] De la Serna, J., Montesinos, P. and Vellenga, E. (2008) Causes and Prognostic Factors of Remission Induction Failure in Patients with Acute Promyelocytic Leukemia Treated with All-Trans Retinoic Acid and Idarubicin. Blood, 111, 3395-3402. https://doi.org/10.1182/blood-2007-07-100669

[9] Hillestad, L.K. (1957) Acute Promyelocytic Leukemia. Acta Medica Scandinavica, 159, 189-194. https://doi.org/10.1111/j.0954-6820.1957.tb00124.x

[10] Tallman, M.S., Nabhan, C., Feusner, J.H. and Rowe, J.M. (2002) Acute Promyelocytic Leukemia: Evolving Therapeutic Strategies. Blood, 99, 759-767. https://doi.org/10.1182/blood.V99.3.759

[11] https://www.uptodate.com/contents/initial-treatment-of-acute-promyelocytic-leuke mia-in-adults? source=history widget $\# \mathrm{H} 28$

[12] Tallman, M.S. and Altman, J.K. (2009) How I Treat Acute Promyelocytic Leukemia. Blood, 114, 5126-5135. https://doi.org/10.1182/blood-2009-07-216457 http://www.bloodjournal.org/content/114/25/5126?sso-checked=true

[13] Tallman, M.S., Nabhan, C., Feusner, J.H. and Rowe, J.M. (2002) Acute Promyelocytic Leukemia: Evolving Therapeutic Strategies. Blood, 99, 759-767.

https://doi.org/10.1182/blood.V99.3.759 